Tenebrio molitor biomass as inducer of lipases and proteases produced by Paecilomyces fumosoroseus

\title{
Biomasa de Tenebrio molitor como inductor de lipasas y proteasas producidas por Paecilomyces fumosoroseus
}

Cinthia Gómez, Ismael Amaya, Reynaldo de la Cruz-Quiroz, Raúl Rodríguez-Herrera, Cristóbal N. Aguilar*

Group of Bioprocesses. Food Research Department, School of Chemistry. Universidad Autónoma de Coahuila. Unidad Saltillo, Boulevard Venustiano Carranza sin número, Colonia Republica, 25280, Saltillo, Coahuila, México. ${ }^{2}$ Agrobiological Control SA de CV, Culiacán, Sinaloa, México.

*Corresponding author.

E-mail address: cristobal.aguilar@uadec.edu.mx (C. N. Aguilar).

Article history:

Received: 26 January 2017 / Received in revised form: 31 May 2017 / Accepted: 26 June 2017 / Published online: 1 July 2017

https://doi.org/10.29267/mxjb.2017.2.2.142

\begin{abstract}
The entomopathogenic fungi are microorganisms commonly used in biocontrol practices of nematodes, insects, among others. The main mechanism of action of these fungi is the parasite mediated by lytic enzymes, depending of the direct interaction of the microorganism with the substrate inducers. In the present study, we evaluated the influence of eight continuous generations of the fungus Paecilomyces fumosoroseus and the effect of the addition of biomass from Tenebrio molitor into the culture medium for the production of proteases and lipases. The P. fumosoroseus strain showed to be an important producer of proteases and lipases under the present conditions evaluated, which gives it a high potential to act as an active agent in bioinsecticidal products. The production of lipases were maintained at homogeneous levels throughout the generations of $P$. fumosoroseus evaluated, however the production of proteases was influenced, with an increase up to the fourth generation. The highest activity values were 800 and $200 \mathrm{U} / \mathrm{L}$ for lipase and protease, respectively.
\end{abstract}

Keywords: biological control, entomoparasitic activity, lipase, protease.

\section{RESUMEN}

Los hongos entomopatógenos son microorganismos comúnmente usados en prácticas de biocontrol de nematodos, insectos, entre otros. El principal mecanismo de acción de estos 
hongos es el parasitismo mediado por enzimas líticas, las cuales dependen de la interacción directa del microorganismo con sustratos inductores. En el presente estudio se evaluó la influencia de 8 generaciones continuas del hongo Paecilomyces fumosoroseus y el efecto de la adición de biomasa de Tenebrio molitor en el medio de cultivo para la producción de enzimas proteasas y lipasas. La cepa de P. fumosoroseus demostró ser un importante productor de proteasas y lipasas bajo las condiciones evaluadas, lo que le confiere alto potencial para actuar como agente activo en productos bioinsecticidas.

La producción de lipasas mantuvo niveles homogéneos a través de las generaciones de $P$. fumosoroseus evaluadas, sin embargo la producción de proteasas si fue influenciada, con un incremento hasta la cuarta generación. Los valores de mayor actividad fueron de 800 y 200 U/L para lipasa y proteasa, respectivamente.

Palabras clave: Actividad entomopatógena, control biológico, lipasa, proteasa.

\section{INTRODUCCION}

Aproximadamente un $40 \%$ del rendimiento total de la producción de alimentos se pierde por efecto de plagas y enfermedades (Chandler et al., 2011). Por lo tanto, el tema de control de plagas que atacan los cultivos alimentarios sigue siendo un gran reto. Existe poco criterio y un uso desmedido de los pesticidas químicos que tradicionalmente se aplican, lo que representa un enorme riesgo a la salud humana, contaminación ambiental y generación de plagas resistentes (John \& Jeeva 2014). Una de las alternativas que en años recientes ha generado gran expectativa es la utilización de organismos benéficos para el control de plagas alimentarias (Glare et al., 2016). En este tópico denominado 'Biocontrol' existen relevantes investigaciones con respecto al uso de bacterias, insectos y hongos con capacidades para controlar plagas de interés agronómico mundial (De La Cruz-Quiroz et al., 2011, Sarwar 2015). En particular los hongos del genero Metarhizium, Beauveria y Trichoderma son algunos de los más estudiados como biopesticidas (De los SantosVillalobos et al., 2012, Loera-Corral et al., 2016, Mascarin \& Jaronski 2016).

Los hongos filamentosos han ganado un gran interés debido los mecanismos de acción que presentan, como lo son: la competencia espacio y nutrientes, antibiosis y actividad parasítica, los cuales en sinergia les confieren gran eficacia contra un amplio rango de organismos plaga (Junaid et al., 2013). El parasitismo es uno de los mecanismos más efectivos que tienen los hongos filamentosos para atacar a sus enemigos, debido a la producción de enzimas líticas que les permiten degradar la pared celular de hongos, insectos y nematodos para utilizarlos como fuente de carbono, causando su muerte (Holden et al., 2003, Markovich \& Kononova 2003, Tondje et al., 2007). El microorganismo parasitante en el biocontol, produce enzimas como celulasas, quitinasas, proteasas y lipasas que han sido asociadas a la degradación de las estructuras tisulares de insectos, nematodos y hongos patógenos.

Algunas especies de Paecilomyces son importantes en el área de biocontrol, ya que es reportado seguro para el uso en cultivos para consumo humano y de bajo impacto ambiental. Es un género de microorganismos entomopatógenos muy atractivo para el manejo integrado de insectos plaga, principalmente debido a las investigaciones donde 
muestran inhibición y eliminación de nematodos e insectos (Abbas et al., 2016, Dong et al., 2016). Además, Paecilomyces es un productor de una serie de enzimas importantes como celulasas, xilanasas, proteasas, quitinasas, amilasas, entre otras (Herrera Bravo de Laguna et al., 2015, Homthong et al., 2016). El objetivo principal de la presente investigación estuvo enfocado en la determinación de la influencia de ocho generaciones continuas del hongo Paecilomyces fumosoroseus y el efecto de la adición de biomasa de Tenebrio molitor en el medio de cultivo para la producción de enzimas proteasas y lipasas.

\section{MATERIALES Y METODOS}

\subsection{Organismos y condiciones de cultivo}

La cepa fúngica de Paecilomyces fumosoroseus y el insecto Tenebrio molitor, fueron proporcionados por el Centro de Microbiología Aplicada (CEMAP) de la empresa Agrobiological Control SA de CV. La cual se conservó en refrigeración $\left(4{ }^{\circ} \mathrm{C}\right)$ en agar Sabouraud -Dextrosa-Levadura (SDY). El hongo fue reactivado sobre placas de Petri con SDY a $25-28{ }^{\circ} \mathrm{C}$ durante 7 días.

\subsection{Activación y cosecha de esporas de $P$. fumosoroseus}

Larvas de T. molitor se sumergieron durante 30 segundos en una suspensión de esporas de $P$. fumosoroseus a una concentración de $1 \times 10^{7}$ conidios/ $\mathrm{mL}$. Después fueron colocadas en cajas de Petri con la dieta recomendada (ABC SA. de CV.) llevando a cabo un monitoreo constante hasta observar la mortalidad. Al final de la prueba, las larvas muertas con desarrollo de micelio sobre la superficie fueron colocadas en una caja de Petri con agar SDY. Las esporas de P. fumosoroseus fueron recuperadas del interior del insecto. Se utilizó el método de aislamiento por siembra por estría en placa para obtener un cultivo monosporico activado. Las cajas de Petri fueron incubadas a $25-28{ }^{\circ} \mathrm{C}$ durante tres días, para después se resembró un explante sobre placas con agar SDY e incubadas nuevamente. Se recuperaron las esporas de $P$. fumosoroseus mediante el raspado de la superficie del agar y colocadas en un desecador. Una semana después se colocaron las esporas en un frasco y se almacenaron en refrigeración $\left(4^{\circ} \mathrm{C}\right)$. A partir de esta etapa, se obtuvieron 8 generaciones consecutivas de $P$. fumosoroseus mediante resiembra continua y evaluadas para la producción enzimática.

\subsection{Análisis proximal de la composición química de la larva de $T$. molitor}

Se llevó a cabo la caracterización química de las larvas de T. molitor por medio de los siguientes análisis: azúcares totales (Dubois et al., 1956), cuantificación de proteínas, lípidos, fibra cruda, materia seca total, humedad y cenizas (AOAC 1990).

\subsection{Cultivo de $P$. fumosoroseus en medio líquido}

Las sales que componen el medio mínimo Czapek-Dox $\left(\mathrm{NaNO}_{3} 0.25 \mathrm{~g}, \mathrm{KH}_{2} \mathrm{PO}_{4} 0.10 \mathrm{~g}\right.$, $\mathrm{MgSO}_{4} 0.05 \mathrm{~g}, \mathrm{KCl} 0.05 \mathrm{~g}$ ) fueron usadas en la formulación del medio de cultivo. La fuente de carbono fue sustituida por biomasa de T. molitor, agregando $2 \mathrm{~g}$ de biomasa por $100 \mathrm{~mL}$ de medio de cultivo. Se utilizó una concentración de $1 \times 10^{6}$ conidios/ $\mathrm{mL}$ para la 
inoculación del medio de cultivo. La incubación fue a $25-28{ }^{\circ} \mathrm{C}$ durante 5 días en agitación constante de $200 \mathrm{rpm}$. El extracto enzimático fue obtenido separando la biomasa del cultivo mediante filtración a través de papel Whatman ${ }^{\circledR}$ No. 1 y después en membrana Millipore ${ }^{\circledR}$ $0.22 \mu \mathrm{m}$.

\subsection{Determinación de las actividades enzimáticas}

La actividad proteasa fue estimada usando el sustrato cromogénico Azocoll (Knox \& Kennedy 1988). Se colocaron $10 \mathrm{mg}$ de Azocoll en un tubo de ensayo, después de agregaron $1 \mathrm{~mL}$ de buffer de acetatos (0.2 M, pH 5.5.), $3.9 \mathrm{~mL}$ de agua y $0.1 \mathrm{~mL}$ del extracto enzimático. Esta mezcla se incubó en agitación a $37{ }^{\circ} \mathrm{C}$ durante 30 minutos. La reacción enzimática se detuvo al filtrar la mezcla por una membrana Millipore ${ }^{\circledR}$ de 0.22 $\mu \mathrm{m}$. La absorbancia se determinó a una longitud de onda de $520 \mathrm{~nm}$. Una unidad enzimática proteasa se definió como la cantidad de enzima necesaria para generar un cambio en la absorbancia de 0.01 por minuto. La actividad lipasa se determinó por espectrometría usando $p$-nitrofenil palmitato (p-NPP) como sustrato. Las muestras se incubaron a $37{ }^{\circ} \mathrm{C}$ durante $20 \mathrm{~min}$. La absorbancia se determinó a una longitud de onda de $348 \mathrm{~nm}$ cada 5 min (Gupta et al., 2002). Una unidad enzimática lipasa se definió como la cantidad de enzima necesaria para liberar $1 \mu \mathrm{mol}$ de $p$-NPP por minuto.

\section{RESULTADOS}

\subsection{Análisis proximal de la composición química de $T$. molitor}

El análisis químico proximal de la biomasa de T. molitor (Tabla 1) muestra que el insecto estaba conformado en su mayoría por azúcares totales y lípidos con un $39.16 \%$ y $38.45 \%$, respectivamente. Sin embargo, las proteínas representaron un importante porcentaje en la composición.

Tabla 1. Composición química de la biomasa de T. molitor.

\begin{tabular}{cc}
\hline Moléculas & Concentración $(\%)$ \\
\hline Materia seca total & $96.16 \pm 0.181$ \\
Azúcares totales & $39.62 \pm 1.022$ \\
Lípidos totales & $38.45 \pm 0.954$ \\
Proteínas & $23.31 \pm 0.563$ \\
Humedad & $3.84 \pm 0.062$ \\
Cenizas & $3.16 \pm 0.074$ \\
\hline
\end{tabular}

\subsection{Determinación de las actividades enzimáticas}

La evaluación de las 8 generaciones de $P$. fumosoroseus permitió la observación de un patrón de producción durante el estudio. Los valores de actividad lipasa resultaron con cierto grado de homogeneidad, a diferencia de la actividad proteasa la cual incrementó 
ligeramente. La actividad lipasa mostró valores en un rango desde 400 a 800 U/L y la actividad proteasa alcanzó valores de hasta 200 U/L (Figura 1).

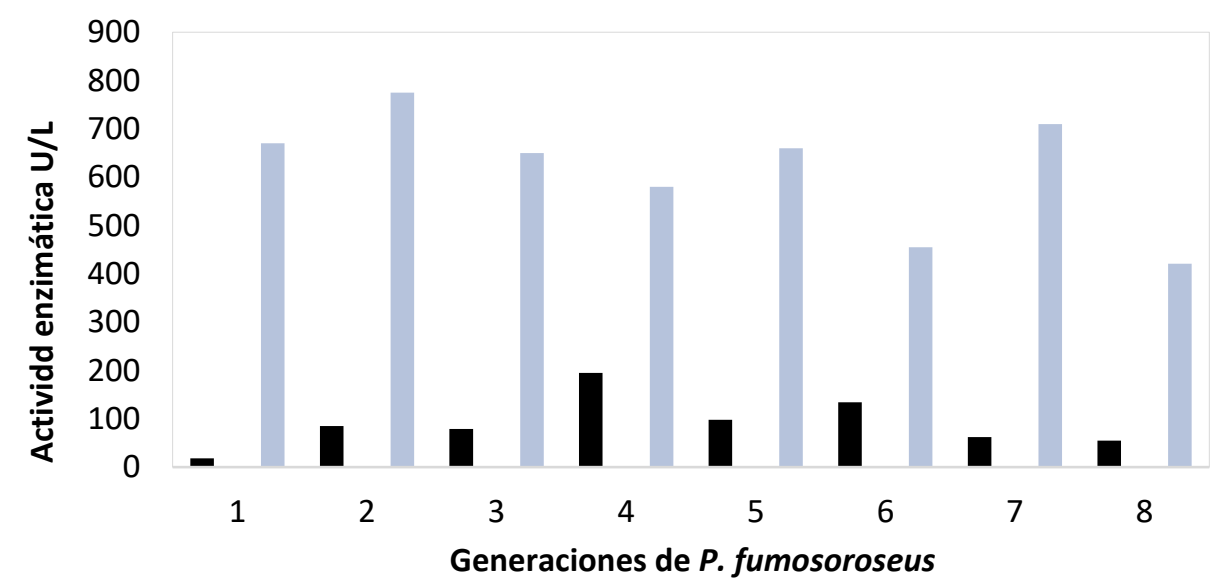

Fig. 1. Actividades enzimáticas de $P$. fumosoroseus usando biomasa de $T$. molitor como sustrato inductor (Lipasa $\square$ y proteasa $\square$ ).

\section{DISCUSIONES}

La actividad entomopatogénica de los hongos filamentosas sobre insectos plaga depende en gran medida de la composición química del mismo, la cual está íntimamente relacionada con la edad y/o estadía del insecto. Es decir, a mayor edad la cutícula se vuelve cada vez más dura, por lo que es mejor atacar una cutícula joven más suave y que por lo tanto será más fácil de degradar (Beris et al., 2013). La degradación de la pared celular es el mecanismo más eficiente que muestran los hongos entomopatógenos mediante la producción de enzimas con poder lítico (Moore \& Prior 1993, Khan et al., 2004, Montesinos-Matías et al., 2011). Por lo tanto las enzimas líticas son comúnmente producidas y secretadas para llevar a cabo la degradación de huevecillos y de la cutícula de nematodos, así como de insectos, esencialmente constituidos por proteínas y quitina asociados con lípidos y compuestos fenólicos (Khan et al., 2004, Castrillo et al., 2005). Particularmente, T. molitor es un insecto que en su estado larvario está compuesto por altos porcentajes de lípidos y proteínas, con valores superiores al 35 y $45 \%$, respectivamente (Samuels \& Paterson 1995). En el presente estudio, las larvas de T. molitor resultaron con una composición con altos niveles de lípidos, valor superior comparándolo con el $32 \%$ reportado por Paul et al., (2017). Niveles bajos de proteínas y valores altos de carbohidratos fueron observados bajo las condiciones de determinación, los cuales difieren a lo reportado en literatura (Kim et al., 2017). Un dato importante es que la composición de la biomasa de T. molitor depende mayormente por el tipo de dieta suministrada, por lo que esta puede ser la principal variación en los datos de la composición química proximal, así como a las condiciones medioambientales del crecimiento del insecto. De acuerdo con Khan et al., (2004), las principales actividades enzimáticas que participan como responsables en el control biológico sobre insectos plaga son las proteasas y lipasas. Es por eso que fueron las dos actividades enzimáticas evaluadas en el presente estudio. A pesar de haber detectado baja concentración de proteína en la biomasa de T. molitor, esta fue suficiente para inducir la producción de proteasas. La actividad proteasa determinada a partir de la generación 1 
del extracto de $P$. fumosoroseus resulta ser un valor muy bajo, sin embargo este valor incrementa generación por generación hasta la numero 4 (200 U/L). Luego se mantiene durante dos generaciones más, para después disminuir en la 7 y 8 hasta estar por debajo de las $100 \mathrm{U} / \mathrm{L}$. Este comportamiento sugiere una posible adaptación al tipo de proteínas que componen a $T$. molitor y que están contenidas en el medio de cultivo. La adaptación mencionada en el párrafo anterior podría influir en la capacidad del hongo para detectar la proteína como sustrato y activar la producción de proteasas específicas. La lipasa mostró mayores niveles de actividad enzimática en el extracto evaluado a través de las 8 generaciones de $P$. fumosoroseus, siempre estando por arriba de las $600 \mathrm{U} / \mathrm{L}$ exceptuando la generación 6 y 8 (entre 400 y 500 U/L, aproximadamente). La evaluación de diferentes generaciones activadas en biomasa de T. molitor mostró un valor de actividad lipasa alto, sin embargo no hubo un incremento de actividad a partir de la generación 1 hasta la 8. Lo que sugiere que el mecanismo de producción de lipasas pudiera estar expresado a su máximo nivel desde la primera generación sin importar si hay subsecuentes activaciones. Las enzimas evaluadas en el presente reporte pueden facilitar la penetración física, la digestión de la composición de las estructuras del hospedero, por lo que se mejoraría la eficiencia de la infección en insectos plaga, ya sea por incremento de la virulencia o por el aumento en la velocidad de infección. Esto demuestra que las enzimas degradadoras son determinantes en el nivel de patogenicidad para la mayoría de los hongos entomopatógenos. Es por eso que actualmente existe un enfoque especial relacionado con la producción de enzimas líticas y este tipo de hongos, debido a los altos rendimientos enzimáticos producidos. El género Paecilomyces tiene amplios reportes en este mismo tópico que van desde producciones exploratorias $\mathrm{u}$ optimizaciones evaluando cepas, sustratos, condiciones de cultivo, etc. (Soares et al., 2010). En particular, la actividad entomopatógena de $P$. fumosoroseus ha sido evaluada en contra de una serie de insectos en donde comúnmente ha mostrado buenos resultados importantes en cuestión de control y mortalidad. Saito \&Aoki (1983), reportaron la evaluación de P. fumosoroseus en contra de Hyphantria cunea y Bombix mori. En ese trabajo los autores correlacionan la actividad lipasa determinada con el tipo de ácido graso presente en el insecto. Años después Ali et al., (2009), reportaron la evaluación de P. fumosoroseus en contra del insecto plaga Plutella xylostella, al causó la muerte en menos de dos días. En ese estudio los autores mencionan que tal efecto fue correlacionado con la cepa fúngica que mostró mayor actividad lipasa. $T$. molitor es un insecto comúnmente utilizado para la evaluación de actividad entomopatógena de un microorganismo, como lo reportado por Montesinos-Matías et al., (2011), quienes detectaron altos índices de virulencia de Beauveria bassiana y desarrollo de biomasa sobre T. molitor asociados con la producción de las enzimas líticas como quitinasas, proteasas y lipasas. Los resultados encontrados en literatura coinciden en que las enzimas lipasas y proteasas son determinantes en el impacto y patogenicidad de Paecilomyces fumosoroseus con un rol de virulencia establecido hacia los insectos hospederos. En el presente estudio la cepa de $P$. fumosoroseus evaluada demuestra ser un hongo filamentoso que produce importantes niveles de proteasas y lipasas utilizando la biomasa de la larva de T. molitor. Es decir que tiene el potencial para ser evaluado o utilizado como agente activo en productos de control biológico, más preciso como bioinsecticida. La producción de lipasas no es afectada por el número de generaciones de $P$. fumosoroseus, es decir bajo las condiciones de producción se mantiene estable, sin embargo es importante considerar que los microorganimos pueden presentar pérdida de actividad o pérdida de virulencia al ser reproducidos de manera industrial bajo condiciones artificiales. 
En cambio, la producción de proteasas si es influenciada por generación. Dentro de algunas de las perspectivas que se tienen para futuras investigaciones se encuentra la evaluación y determinación de dosis letales, condiciones de aplicación, producción de biomasa de $P$. fumosoroseus, escalamiento de producción, vida de anaquel, viabilidad y efectividad.

\section{AGRADECIMIENTOS}

Los autores agradecen el apoyo financiero otorgado por el Consejo Nacional de Ciencia y Tecnología y de la Empresa Agrobiological Control SA de CV.

\section{CONFLICTO DE INTERES}

Los autores declaran que no existe conflicto de intereses

\section{REFERENCIAS}

Abbas H., Javed N., Khan SA., Kamran M. \& Atiq M. 2016. Exploitation of Nematicidal Potential of Paecilomyces lilacinus against Root Knot Nematode on Eggplant. International Journal of Vegetable Science. 22(1): 85-90.

Ali S., Huang Z. \& Ren S. 2009. Media composition influences on growth, enzyme activity, and virulence of the entomopathogen hyphomycete Isaria fumosoroseus. Entomologia Experimentalis et Applicata. 131(1): 30-38.

AOAC. 1990. Official Methods of Analysis. . 15 th Ed. K. Erlich (Ed.). Arlington, Virginia, USA. . 59-87: 1049-1106.

Beris EI., Papachristos DP., Fytrou A., Antonatos SA. \& Kontodimas DC. 2013. Pathogenicity of three entomopathogenic fungi on pupae and adults of the Mediterranean fruit fly, Ceratitis capitata (Diptera: Tephritidae). Journal of Pest Science. 86(2): 275-284.

Castrillo LA., Roberts DW. \& Vandenberg JD. 2005. The fungal past, present, and future: Germination, ramification, and reproduction. Journal of Invertebrate Pathology. 89(1): 4656.

Chandler D., Bailey AS., Tatchell GM., Davidson G., Greaves J. \& Grant WP. 2011. The development, regulation and use of biopesticides for integrated pest management. Philosophical Transactions of the Royal Society B: Biological Sciences. 366(1573): 19871998.

De La Cruz-Quiroz R., Chavez C., Hernandez M., Rodriguez R., Hernandez D. \& Aguilar C. 2011. Antagonist capacity of newly isolated strains of Pseudomonas Fluorescens against three important phytopathogenic bacteria .American Journal of Agricultural and Biological Sciences. 6(2): 267-272.

De los Santos-Villalobos S., Hernández-Rodríguez LE., Villaseñor-Ortega F. \& PeñaCabriales JJ. 2012. Production of Trichoderma asperellum T8a spores by a "home-made" solid-state fermentation of mango industrial wastes. BioResources. 7(4): 4938-4951. 
Dong T., Zhang B., Jiang Y. \& Hu Q. 2016. Isolation and Classification of Fungal Whitefly Entomopathogens from Soils of Qinghai-Tibet Plateau and Gansu Corridor in China. PLOS ONE. 11(5): e0156087.

Dubois M., Gilles K., Hamilton J., Rebers P. \& Smith F. 1956. Colorimetric method for determination of sugars and related substances. Analytical Chemistry. 28(3): 350-356.

Glare TR., Gwynn RL. \& Moran-Diez ME. 2016. Development of Biopesticides and Future Opportunities. In Microbial-Based Biopesticides: Methods and Protocols. 211-221.

Gupta N., Rathi P. \& Gupta R. 2002. Simplified para-nitrophenyl palmitate assay for lipases and esterases. Analytical Biochemistry. 311(1): 98-99.

Herrera Bravo de Laguna I., Toledo Marante FJ. \& Mioso R. 2015. Enzymes and bioproducts produced by the ascomycete fungus Paecilomyces variotii. Journal of Applied Microbiology. 119(6): 1455-1466.

Holden HM., Rayment I. \& Thoden JB. 2003. Structure and function of enzymes of the Leloir pathway for galactose metabolism. J Biol Chem. 278.

Homthong M., Kubera A., Srihuttagum M. \& Hongtrakul V. 2016. Isolation and characterization of chitinase from soil fungi, Paecilomyces sp. Agriculture and Natural Resources. 50(4): 232-242.

John NS. \& Jeeva ML. 2014. Efficacy of cassava by-products as carrier materials of Trichoderma harzianum, a biocontrol agent against Sclerotium rolfsii causing collar rot in elephant foot yam. Journal of Root Crops. 40(1): 1-6.

Junaid JM., Dar NA., Bhat TA., Bhat AH. \& Bhat MA. 2013. Commercial Biocontrol Agents and Their Mechanism of Action in the Management of Plant Pathogens. International Journal of Modern Plant Animal Sciences. 1(12): 39-57.

Khan A., Williams KL. \& Nevalainen HKM. 2004. Effects of Paecilomyces lilacinus protease and chitinase on the eggshell structures and hatching of Meloidogyne javanica juveniles. Biological Control. 31(3): 346-352.

Kim S-K., Weaver CM. \& Choi M-K. 2017. Proximate composition and mineral content of five edible insects consumed in Korea. CyTA - Journal of Food. 15(1): 143-146.

Knox DP. \& Kennedy MW. 1988. Proteinases released by the parasitic larval stages of Ascaris suum, and their inhibition by antibody. Molecular and Biochemical Parasitology. 28(3): 207-216.

Loera-Corral O., Porcayo-Loza J., Montesinos-Matias R. \& Favela-Torres E, Production of conidia by the fungus Metarhizium anisopliae using solid-state fermentation. In MicrobialBased Biopesticides: Methods and Protocols, T.R. Glare and M.E. Moran-Diez, Editors. 2016, Springer New York: New York, NY. p. 61-69. 
Markovich N. \& Kononova G. 2003. Lytic Enzymes of Trichoderma and Their Role in Plant Defense from Fungal Diseases: A Review. Applied Biochemistry and Microbiology. 39(4): 341-351.

Mascarin GM. \& Jaronski ST. 2016. The production and uses of Beauveria bassiana as a microbial insecticide. World Journal of Microbiology and Biotechnology. 32(11): 177.

Montesinos-Matías R., Viniegra-González G., Alatorre-Rosas R. \& Loera O. 2011. Relationship between virulence and enzymatic profiles in the cuticle of Tenebrio molitor by 2-deoxy-d-glucose-resistant mutants of Beauveria bassiana (Bals.) Vuill. World Journal of Microbiology and Biotechnology. 27(9): 2095-2102.

Moore D. \& Prior C. 1993. The potential of mycoinsecticides. Biocontrol News and Information. 14: 31-40.

Paul A., Frederich M., Megido RC., Alabi T., Malik P., Uyttenbroeck R., Francis F., Blecker C., Haubruge E., Lognay G. \& Danthine S. 2017. Insect fatty acids: A comparison of lipids from three Orthopterans and Tenebrio molitor L. larvae. Journal of Asia-Pacific Entomology. 20(2): 337-340.

Saito T. \& Aoki J. 1983. Toxicity of free fatty acids on the larval surfaces of two lepidopterus insects towards Beauveria bassiana (Bals.) Vuill. and Paecilomyces fumosoroseus (Wize) Brown et Smith (Deuteromycetes: Moniliales). Applied Entomology and Zoology. 18(2): 225-233.

Samuels R. \& Paterson I. 1995. Cuticle degrading proteases from insect moulting fluid and culture filtrates of entomopathogenic fungi. Comparative Biochemistry and Physiology Part B: Biochemistry and Molecular Biology. 110(4): 661-669.

Sarwar M. 2015. Biopesticides : An Effective and Environmental Friendly Insect-Pests Inhibitor Line of Action. International Journal of Engineering and Advanced Research Technology. 1(2): 10-15.

Soares F., Braga F., Geniêr H., de Araújo J., Ferreira S., Araujo J., Tavela A., Vilela V. \& de Queiróz J. 2010. Optimization of medium composition for protease production by Paecilomyces marquandii in solid-state-fermentation using response surface methodology. African Journal of Microbiology Research. 4 (24): 2699-2703.

Tondje P., Roberts D., Bon M., Widmer T., Samuels G., Ismaiel A., Begoude A., Tchana T., Nyemb-Tshomb E., Ndoumbe-Nkeng M., Bateman R., Fontem D. \& Hebbar K. 2007. Isolation and identification of mycoparasitic isolates of Trichoderma asperellum with potential for suppression of black pod disease of cacao in Cameroon. Biological Control. 43(2): 202-212. 